\title{
VOLUME XXXII
}

\section{INDEX OF PAPERS BY AUTHORS}

Author

ANSHEL, MICHAEL

Title

Page

Vector Groups and the Equality Problem for Vector

Addition Systems

BABUŠKKA, I. \& OSBORN, J.E. Numerical Treatment of Eigenvalue Problems for Differ-

ential Equations with Discontinuous Coefficients ..........

BANEGAS, ALEXANDRA

Fast Poisson Solvers for Problems with Sparsity

Linear Chebyshev Approximation of Complex-Valued Functions

BARRODALE, I., DELVES, L. M. \& MASON, J.C.

BARROW, DAVID L.

On Multiple Node Gaussian Quadrature Formulae

Unicity of Best Mean Approximation by Second Order

BARROW, D. L., CHUI, C.K., SMITH, P.W. \& WARD, J.D,

BAYLISS, ALVIN

Splines with Variable Knots

A Double Shooting Scheme for Certain Unstable and

Singular Boundary Value Problems

BAYS, CARTER \& HUDSON, RICHARD $H$.

On the Fluctuations of Littlewood for Primes of the Form $4 n \pm 1$

BAYS, CARTER \& HUDSON, RICHARD $H$.

BENNETT, J. M.

Details of the First Region of Integers $x$ with $\pi_{3,2}(x)<$ $\pi_{3,1}(x)$

See: FRASER, W. \& BENNETT, J. M

BENTON, T.C. \& KNOBLE, H. D.

BLAIR, J.M., EDWARDS, C.A. Rational Chebyshev Approximations for the Bickley \& JOHNSON, J.H. BOYD, DAVID W.

BRAMBLE, JAMES H. \& SCOTT, RIDGWAY

BUNTING, PAUL W., van Deciding Associativity for Partial Multiplication Tables of LEEUWEN, JAN \& TAMARI, DOV

BURNS, ANNE M.

Functions $\mathrm{Ki}_{n}(x)$

Pisot and Salem Numbers in Intervals of the Real Line..... 1244

Simultaneous Approximation in Scales of Banach Spaces ... 947

Order 3

A Necessary Condition for the Stability of a Difference Approximation to a Hyperbolic System of Partial Differential Equations

CASH, J. R.

An Extension of Olver's Method for the Numerical Solution of Linear Recurrence Relations

CHAN, C.

See: RIDDELL, J. \& CHAN, C.

CHIN, R.C. Y. \& HEDSTROM, G.W.

A Dispersion Analysis for Difference Schemes: Tables of Generalized Airy Functions.

CHOW, SHUI-NEE, MALLETPARET, JOHN \& YORKE, JAMES A.

CHRISTIANSEN, J. \& RUSSELL, R. D.

CHUI, C. K.

CIARLET, PHILIPPE G.

Finding Zeroes of Maps: Homotopy Methods That Are Constructive with Probability One

Error Analysis for Spline Collocation Methods with Application to Knot Selection

See: BARROW, D. L., CHUI, C. K., SMITH, P.W. \& WARD, J. D.

Interpolation Error Estimates for the Reduced HsiehClough-Tocher Triangle

A Note on the Operator Compact Implicit Method for the Wave Equation. MOHAMED, JULIE

On de Vogelaere's Method for $y^{\prime \prime}=f(x, y)$ 
Author

DIAZ Y DIAZ, F. DiDONATO, A. R. DOTSON, W. G., JR.
Title

Page

DOUGLAS, JIM, JR., DUPONT, A Quasi-Projection Analysis of Galerkin Methods for TODD \& WHEELER, MARY F.

DOWNS, T.

DREZNER, Z.

DUPONT, TODD

EDWARDS, C. A.

EDWARDS, M. J.

EISENBEIS, H., FREY, G. \& OMMERBORN, B.

ERNVALL, R. \& METSÄNKYLÄ, T.

FATUNLA, SIMEON OLA.

FISCHER, J. \& MCKAY, J.

FRASER, W. \& BENNETT, J. $M$.

FREY, A. E., HALL, C. A. \& PORSCHING, T. A.

FREY, G.

GAFFNEY, P. W.

GALÁNTAI, AURÉL

GAUTSCHI, WALTER

\& SLAVIK, JOSEF

GIROIRE, J. \& NEDELEC, J. C.

GODWIN, H. J.

GOLDBERG, MOSHE \&

TADMOR, EITAN

GORDON, WILLIAM J. \& WIXOM, JAMES A.

GREENSTADT, JOHN GRINSTEAD, CHARLES M. GUPTA, G. K.

\section{GUPTA, HANSRAJ \\ HADJIDIMOS, APOSTOLOS \\ HALD, OLE \& MAUCERI DEL PRETE, VINCENZA HALD, OLE H.}

HALL, C. A. HARTEN, AMIRAM

HEDSTROM, G. W. HENDY, M. D. HICKS, D. L.
On Some Families of Imaginary Quadratic Fields

An Approximation for $\int_{x}^{\infty} e^{-t 2 / 2} t^{p} d t, x>0, p$ real

An Iterative Process for Nonlinear Monotonic Nonexpansive Operators in Hilbert Space.

Parabolic and Hyperbolic Equations

637

271

223

345

See: O'NEILL, C. J. \& DOWNS, T.

1144

Computation of the Bivariate Normal Integral .................. 277

See: DOUGLAS, JIM, JR., DUPONT, TODD \& WHEELER, MARY F.

345

See: BLAIR, J. M., EDWARDS, C. A. \& JOHNSON, J.H.. 876

See: ZAKIAN, V. \& EDWARDS, M. J.

Computation of the 2-Rank of Pure Cubic Fields

519

559

Cyclotomic Invariants and E-Irregular Primes.

617

An Implicit Two-Point Numerical Integration Formula for Linear and Nonlinear Stiff Systems of Ordinary Differential Equations

The ${ }^{\prime}$ Nonabelian Simple Groups $G,|G|<10^{6}$-Maximal Subgroups.

A Method of Virtual Displacements for the Degenerate Discrete $l_{1}$ Approximation Problem ... 1293

Some Results on the Global Inversion of Bilinear and Quadratic Isoparametric Finite Element Transformations

See: EISENBEIS, H., FREY, G. \& OMMERBORN, B........

To Compute the Optimal Interpolation Formula .................

The Comparison of Numerical Methods for Solving Polynomial Equations.

On the Computation of Modified Bessel Function Ratios...

Numerical Solution of an Exterior Neumann Problem Using a Double Layer Potential

A Note on Congruent Numbers

Scheme-Independent Stability Criteria for Difference Approximations of Hyperbolic Initial-Boundary Value Problems. I

Shepard's Method of "Metric Interpolation" to Bivariate and Multivariate Interpolation

Revision of a Derivative-Free Quasi-Newton Method ......... 201

On a Method of Solving a Class of Diophantine Equations. 936

Implementing Second-Derivative Multistep Methods Using the Nordsieck Polynomial Representation..................... 13

Finite Differences of the Partition Function...................... 1241

Convergence of Vortex Methods for Euler's Equations........ 791

The Inverse Sturm-Liouville Problem and the Rayleigh-

See: FREY, A. E., HALL, C. A. \& PORSCHING, T. A. .... 725

The Artificial Compression Method for Computation of Shocks and Contact Discontinuities: III. Self-Adjusting Hybrid Schemes.

See: CHIN, R. C. Y. \& HEDSTROM, G. W. ...................... 1163

See: JEANS, N. S. \& HENDY, M. D.

Stability Analysis of WONDY (A Hydrocode Based on the the Artificial Viscosity Method of von Neumann and Richtmyer) for a Special Case of Maxwell's Law
Accelerated Overrelaxation Method .................................. 149 Ritz Method 
HOLTE, R.

HOUSTIS, E. N. \&

See: WILLIAMS, H. C. \& HOLTE, R.

PAPATHEODOROU, T.S.

A Collocation Method for Fredholm Integral Equations of the Second Kind

HOWLAND, JAMES LUCIEN

The Resultant Iteration for Determining the Stability of a Polynomial

HUDSON, RICHARD H.

See: BAYS, CARTER \& HUDSON, RICHARD H.

JEANS, N. S. \& HENDY, M. D. Determining the Fundamental Unit of a Pure Cubic Field Given Any Unit

JELTSCH, ROLF

JOHNSON, J. H. JOHNSON, WELLS

Complete Characterization of Multistep Methods with an Interval of Periodicity for Solving $y^{\prime \prime}=f(x, y) \ldots \ldots \ldots . . . .1108$

See: BLAIR, J. M., EDWARDS, C. A. \& JOHNSON, J. H. 876

KAUFMAN, EDWIN H., JR., LEEMING, DAVID J. \&

TAYLOR, G. D.

KEARFOTT, BAKER

KELLER, H. B. \& PEREYRA, $V$.

KELLOGG, R. BRUCE \& TSAN, ALICE

KING, J. THOMAS \& SERBIN, STEVEN M.

KISHORE, MASAO

KNOBLE, H. D.

KRAMARZ, LUIS

LAM, BINH

LEEMING, DAVID J.

van LEEUWEN, JAN

LEHMER, D. H. \& MASLEY, J. $M$.

LEVENTHAL, STEPHEN H.

LING, CHIH-BING

MCALLISTER, DAVID F. \& ROULIER, JOHN A. MCCABE, J. H.

McDONOUGH, JAMES

McKAY, J.

MAJDA, ANDREW, MCDONOUGH, JAMES \& OSHER, STANLEY MALLET-PARET, JOHN

MANSFIELD, LOIS

MASLEY, J. M.

MASON, J. C.

MAUCERI DEL PRETE, VINCENZA

METSÄNKYLÄ, T.

A Combined Remes-Differential Correction Algorithm for Rational Approximation.

A Proof of Convergence and an Error Bound for the Method of Bisection in $\mathbf{R}^{n}$

Symbolic Generation of Finite Difference Formulae ......... 955

Analysis of Some Difference Approximations for a Singular Perturbation Problem Without Turning Points

Computational Experiments and Techniques for the Penalty Method with Extrapolation

Odd Integers $N$ With Five Distinct Prime Factors for Which $2-10^{-12}<\sigma(N) / N<2+10^{-12}$

See: BENTON, T. C. \& KNOBLE, H. D.

Global Approximations to Solutions of Initial Value Problems

On the Convergence of a Quasi-Newton Method for Sparse Nonlinear Systems

See: KAUFMAN, EDWIN H., JR., LEEMING, DAVID J. \& TAYLOR, G. D.

See: BUNTING, PAUL W., van LEEUWEN, JAN \& TAMARI, DOV

Table of the Cyclotomic Class Numbers $h^{*}(p)$ and Their Factors for $200<p<521$.

See: CIMENT, MELVYN \& LEVENTHAL, STEPHEN H. .. 143

Further Evaluation of Howland Integrals ........................ $\quad 900$

Interpolation by Convex Quadratic Splines ..................... 1154

A Further Correspondence Property of $M$ Fractions 1303 See: MAJDA, ANDREW, MCDONOUGH, JAMES \& OSHER, STANLEY

See: FISCHER, J.

The Fourier Method for Nonsmooth Initial Data

See: CHOW, SHUI-NEE, MALLET-PARET, JOHN \& YORKE, JAMES A.

A Clough-Tocher Type Element Useful for Fourth Order Problems Over Nonpolygonal Domains ........................ 135

See: LEHMER, D. H. \& MASLEY, J. M. ......................... 577

See: BARRODALE, I., DELVES, L. M. \& MASON, J. C. .. 853

See: HALD, OLE \& MAUCERI DEL PRETE, VINCENZA

See: ERNVALL, R. \& METSÄNKYLÄ,T. 
Author

MIRANKER, W. L. \& van

VELDHUIZEN, $M$.

MOHAMED, JULIE

MONEGATO, GIOVANNI

MONEGATO, GIOVANNI

NEDELEC, J. C.

NEVANLINNA, OLAVI

NICOLAIDES, R. A.

NICOLAIDES, R. A.

OMMERBORN, B.

O'NEILL, C. J. \& DOWNS, T.

OSBORN, J. E.

OSHER, STANLEY

PAPATHEODOROU, T. S.

PARRY, CHARLES J.

PEREYRA, V.

PEXTON, ROBERT L. \& STEIGER, ARNO D.

POLLARD, J. M.

PORSCHING, T. A.

RAKOTCH, E.

RIDDELL, J. \& CHAN, C.

ROULIER, JOHN A.

ROWEN, LOUIS HALLE \&

SCHILD, URI

RUSSELL, R. D.

SCHATZ, A. H. \&

WAHLBIN, L. B.

SCHILD, URI

SCHMIDT, PAUL W.

SCHMIDT, PAUL W.

SCHNABEL, ROBERT B.

SCHONFELDER, J. L.

SCOTT, RIDGWAY

SERBIN, STEVEN M.

SHAMPINE, L. F.

SHANTARAM, R.

SHIPPEE, D. E.

SLAVIK, JOSEF

SMITH, P. W.

SMYTH, W. F.

STEIGER, ARNO D.

STEVENS, R. S. \&

SHANTARAM, R.

STOTHERS, W. W.

TADMOR, EITAN
Title

Page

The Method of Envelopes

453

See: COLEMAN, JOHN P. \& MOHAMED, JULIE 751

Positivity of the Weights of Extended Gauss-Legendre Quadrature Rules.

Some Remarks on the Construction of Extended Gaussian Quadrature Rules.

247

See: GIROIRE, J. \& NEDELEC, J. C.

On the Convergence of Difference Approximations to Nonlinear Contraction Semigroups in Hilbert Spaces.

On the Observed Rate of Convergence of an Iterative Method Applied to a Model Elliptic Difference Equation

On Multigrid Convergence in the Indefinite Case ............... 1082

See: EISENBEIS, H., FREY, G. \& OMMERBORN, B. ...... 559

A Numerical Accuracy Consideration in Polynomial Deflation

See: BABUŠKKA, I. \& OSBORN, J. E................................. 991

See: MAJDA, ANDREW, MCDONOUGH, JAMES \& OSHER, STANLEY

See: HOUSTIS, E. N. \& PAPATHEODOROU, T. S. ......... 159

On the Class Number of Relative Quadratic Fields ........... 1261

See: KELLER, H. B. \& PEREYRA, V. .............................. 955

Roots of Two Transcendental Equations as Functions of a Continuous Real Parameter

Monte Carlo Methods for Index Computation $(\bmod p)$..... 918

See: FREY, A. E., HALL, C. A. \& PORSCHING, T. A..... 725

Improved Error Estimates for Numerical Solutions of

Symmetric Integral Equations

Some Extremal 2-Bases ................................................. 630

See: McALLISTER, DAVID F. \& ROULIER, JOHN A. .... 1154

A Scalar Expression for Matrices With Symplectic Involution

See: CHRISTIANSEN, J. \& RUSSELL, R. D.

Maximum Norm Estimates in the Finite Element Method on Plane Polygonal Domains. Part 1 ........................... 73

See: ROWEN, LOUIS HALLE \& SCHILD, URI ................ 607

Evaluation of the Integral

$$
\int_{0}^{\infty}\left(t^{2 \alpha-1} J_{\nu}\left(x \sqrt{1+t^{2}}\right) /\left(1+t^{2}\right)^{\alpha+\beta-1}\right) d t
$$

An Asymptotic Approximation for a Type of Fourier Integral

Minimum Norm Symmetric Quasi-Newton Updates

Restricted to Subspaces

Chebyshev Expansions for the Error and Related Functions

See: BRAMBLE, JAMES H. \& SCOTT, RIDGWAY

See: KING, J. THOMAS \& SERBIN, STEVEN M.

Limiting Precision in Differential Equation Solvers. II:

Sources of Trouble and Starting a Code

See: STEVENS, R. S. \& SHANTARAM, R. ..................... 635

Four New Factors of Fermat Numbers ............................ 941

See: GAUTSCHI, WALTER \& SLAVIK, JOSEF .............. 865

See: BARROW, D. L., CHUI, C. K., SMITH, P. W. \& WARD, J. D.

The Construction of Rational Iterating Functions ............. 811

See: PEXTON, ROBERT L. \& STEIGER, ARNO D. ........ 511

Computer-Generated van der Waerden Partitions ............... 635

Free Subgroups of the Free Product of Cyclic Groups........ 1274

See: GOLDBERG, MOSHE \& TADMOR, EITAN .............. 1097 
Author

TAKÁCS, LAJOS

TAMARI, DOV

TAYLOR, G. D.

TOINT, PH. L.

TSAN, ALICE

USMANI, RIAZ A.

VAUGHAN, THERESA P.

van VELDHUIZEN, M. WAGSTAFF, SAMUEL S., JR. WAHLBIN, L. B.

WALKER, P. L.

WANG, PAUL $S$.

WARD, J. D.

WATANABE, DANIEL $S$. WHEELER, MARY F.

WILLIAMS, H. C.

WILLIAMS, H. C. \& HOLTE, $R$.

WINOGRAD, $S$.

WIXOM, JAMES A.

WOLKOWICZ, H. \&

ZLOBEC, $S$.

YORKE, JAMES A.

ZAKIAN, V. \& EDWARDS, M. J.

ZASSENHAUS, HANS

ZLÁMAL, MILOŠ

ZLOBEC, S.
Title

Page

A Sum of Binomial Coefficients

1271

See: BUNTING, PAUL W., van LEEUWEN, JAN \&

TAMARI, DOV

593

See: KAUFMAN, EDWIN H., JR., LEEMING, DAVID J.

\& TAYLOR, G. D.

Some Numerical Results Using a Sparse Matrix Updating

Formula in Unconstrained Optimization

839

1025

See: KELLOGG, R. BRUCE \& TSAN, ALICE

Discrete Variable Methods for a Boundary Value Problem with Engineering Applications.

1087

A Generalization of the Simple Continued Fraction

Algorithm

See: MIRANKER, W. L. \& van VELDHUIZEN, M. ........... 453

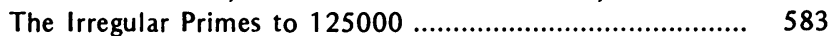

See: SCHATZ, A. H. \& WAHLBIN, L. B.......................... 73

On an Integral Summable to $2 \xi(s) / s(s-1)$...................... 1311

An Improved Multivariate Polynomial Factoring Algorithm

See: BARROW, D. L., CHUI, C. K., SMITH, P. W. \& WARD, J. D...

Block Implicit One-Step Methods 405

See: DOUGLAS, JIM, JR., DUPONT, TODD \& WHEELER, MARY $F$.

Some Primes with Interesting Digit Patterns ....................... 1306

Some Observations on Primality Testing ............................ 905

On Computing the Discrete Fourier Transform ................. 175

See: GORDON, WILLIAM J. \& WIXOM, JAMES A........... 253

Calculating the Best Approximate Solution of an Operator Equation

See: CHOW, SHUI-NEE, MALLET-PARET, JOHN \& YORKE, JAMES A.

Tabulation of Constants for Full Grade ${ }_{M N}$ Approximants

A Remark on the Hensel Factorization Method ................ 287

Superconvergence and Reduced Integration in the Finite

Element Method

See: WOLKOWICZ, H. \& ZLOBEC, S.

1183 


\section{SUBJECT CLASSIFICATION SYSTEM \\ FOR INDEX OF REVIEWS}

The following subject classification system is used for the yearly index of reviews. Individual reviews in the quarterly issues are assigned index classification numbers in simplified form.

1.00 Biography and Bibliography (History)

2.00 Selected Topics in Numerical Analysis

2.05 Approximation Theory

2.05.1 Least Squares, Curve Fitting, Harmonic Analysis

2.05.2 Chebyshev (Best) Approximation

2.05.3 Interpolation, Extrapolation

2.05.4 Inverse Interpolation

2.05.5 Rational Approximation

2.05.6 Splines

2.10 Numerical Integration

2.10.1 One-Dimensional

2.10.2 Multi-Dimensional

2.10.3 Monte Carlo

2.15 Numerical Differentiation

2.20 Roots of tiquations

2.25 Evaluation of Series

2.30 Continued Fractions

2.35 Iteration Methods, Acceleration Techniques

2.40 Differences, Divided Differences

2.45 Algorithms, General Theory

2.50 Inequalities

2.55 Stability of Computation, Significance Arithmetic

2.60 Complexity of Computation

3.00 Linear Algebra

3.05 Matrices

3.10 Linear Equations

3.10.1 Error Analysis

3.15 Eigenvalues and Eigenvectors

3.15.1 Error Analysis

3.20 Matrix Inversion and Pseudo-Inverses

3.20.1 Error Analysis

3.25 Linear and Nonlinear Programming, Theory of Games

3.25.1 Error Analysis

3.30 Determinants

3.35 Sparse Matrices

4.00 Ordinary Differential Equations

4.05 Initial Value Problems

4.05.1 Analytic Methods

4.05.1.1 Error Analysis

4.05.2 One-Step Methods

4.05.2.1 Error Analysis

4.05.3 Multistep Methods

4.05.3.1 Error Analysis

4.10 Linear Boundary Value and Eigenvalue Problems

4.10.1 Analytic Methods

4.10.1.1 Error Analysis

4.10.2 Initial Value (Trial and Error or Shooting) Methods

4.10.2.1 Error Analysis 
4.10.3 Finite Difference (Nonshooting) Methods

4.10.3.1 Error Analysis

4.10.4 Finite Element Methods

4.10.4.1 Error Analysis

4.15 Nonlinear Boundary Value and Eigenvalue Problems

4.15.1 Analytic Methods

4.15.1.1 Error Analysis

4.15.2 Initial Value (Trial and Error or Shooting) Methods

4.15.2.1 Error Analysis

4.15.3 Finite Difference (Nonshooting) Methods

4.15.3.1 Error Analysis

5.00 Partial Differential Equations

5.05 Initial Value Problems

5.05.1 Analytic Methods

5.05.1.1 Error Analysis

5.05.2 Explicit Difference Methods

5.05.2.1 Error Analysis

5.05.3 Implicit Difference Methods, Iterative Schemes 5.05.3.1 Error Analysis

5.05.4 Finite Element and Other Approximation Methods 5.05.4.1 Error Analysis

5.10 Boundary Value Problems

5.10.1 Analytic Methods 5.10.1.1 Error Analysis

5.10.2 Finite Difference Methods, Iterative Schemes 5.10.2.1 Error Analysis

5.10.3 Finite Element and Other Approximation Methods 5.10.3.1 Error Analysis

5.15 Eigenvalue Problems

5.15.1 Analytic Methods

5.15.1.1 Error Analysis

5.15.2 Finite Difference Methods, Iterative Schemes 5.15.2.1 Error Analysis

5.15.3 Finite Element and Other Approximation Methods 5.15.3.1 Error Analysis

5.20 Mixed Initial and Boundary Value Problems

5.20.1 Analytic Methods 5.20.1.1 Error Analysis

5.20.2 Explicit Difference Methods 5.20.2.1 Error Analysis

5.20.3 Implicit Difference Methods, Iterative Schemes 5.20.3.1 Error Analysis

5.20.4 Finite Element and Other Approximation Methods 5.20.4.1 Error Analysis

6.00 Other Functional Equations

6.05 Difference Equations

6.10 Difference-Differential Equations

6.15 Integral Equations

6.20 Integro-Differential Equations

6.25 Convolution Equations

6.30 Variotional Equations

6.35 Abstract Operator Equations

6.40 Stochastic Differential Equations

7.00 Special Functions

7.05 Mathematical Constants, Special Polynomials (Nonorthogonal) and Numbers: Bernoulli, Euler, Stirling, Binomial Coetficients, Factorials

7.10 Elementary Functions, Powers and Roots

7.15 Gamma Functions, Psi Functions, Zeto Functions and Related Functions

7.20 Incomplete Gamma Functions 
7.20.1 Exponential Integral, Cosine and Sine Integrals and Related Functions 7.20.2 Error Functions, Fresnel Integrals and Related Functions

7.25 Confluent Hypergeometric Functions

7.25.1 Parabolic Cylinder Functions

7.25.2 Coulomb Wave Functions

7.30 Bessel Functions

7.35 Lommel Functions, Struve Functions, Anger-Weber Functions and Associated Bessel Functions

7.40 Legendre Functions

7.45 Goussian Hypergeometric Functions

7.50 Orthogonal Polynomials and Functions

7.55 Expansions in Series of Orihogonal Polynomials, Bessel Functions, Other Functions

7.60 Elliptical Integrals and Functions, Weierstrass Elliptic Integrals, Related Functions

7.65 Mathicu Functions

7.70 Spheroidal Wave Functions, Other W'ave Functions

7.75 Generalized Hypergeometric Functions of a Single Variable

7.80 Generalized Hypergeometric Functions of More Than One Variuble

7.85 Busic Hypergeometric Functions

7.90 Integral Transforms

7.95 Numerical Tables of Integrals and Transforms

7.100 Numerical Tables of Infinite Series

7.105 Handbooks of Mathemutical Tables and Formulae

8.00 Probability and Statistics

8.05 Rundom Numbers

8.05.1 Tables

8.10 Monte Carlo, Markou' Chains

8.15 Multivariate Analysis

8.20 Regression Analysis

8.25 Analysis of Variance

8.30 Time Series Analysis

8.35 Nonpurametric Analysis

8.40 Sequential Analysis

8.45 Classical Statistics

8.50 Handbooks of Tables and Formulae

9.00 Number Theory

9.05 Mersenne, Fermat, Perfect and Related Numbers

9.10 Number-Theoretic Functions and Tables

9.15 Binomial Congruences, Primitive Roots, Residues, etc.

9.20 Primes and Their Distribution

9.25 Factorizution

9.30 Forms and Diophantine Equations

9.35 Continued Fractions and Diophantine Approximation

9.40 Normal Numbers and Distribution of Digits

9.45 Modular Computotion

10.00 Algebra and Combinatorial Theory

10.05 Groups, Rings, Fields, Algebras

10.10 Finite Fields

10.15 Irreducible Polynomiuls

10.20 Arrays, Latin Squares

10.25 Projective Planes, Block Designs, Difference Sets

10.30 Permutations, Combinatorial Identities

10.35 Graph Theory

\subsection{Geometry}

11.05 Coordinate Conversion Tables

11.10 Polyhedra and Polytopes

11.15 Graph Theory

12.00 Computers and Other Aids to Computation

12.05 Digital Computers 
12.05.1 Coding, Programming and Software

12.05.2 Design and Hardware

12.05.3 Computer Aided Instruction

12.10 Anolog Computers

12.10.1 Design and Hardware

12.15 Digital-Analog (Hybrid) Computers

12.15.1 Coding, Programming and Software

12.15.2 Design and Hardware

12.20 Mechanical Aids to Computation-Slide Rules, Desk Calculators, Others

12.25 Nomographs

13.00 Applications

13.05 Physical and Chemical Sciences

13.05.1 Weights and Measures Tables

13.10 Astronomy, Astrophysics

13.10.1 Novigation Tables

13.15 Engineering Sciences

13.15.1 Engineering Tables

13.20 Earth Sciences, Atmospheric Sciences, Fluid Dynamics

13.20.1 Triangulation and Geodetic Tables

13.25 Biology and the Behavioral Sciences

13.30 Economics and the Social Sciences

13.35 Information Theory, Automata, Logic Control Theory, Dynamic Programming, Cybernetics

13.40 Management Problems, Dato Analysis and Processing

13.45 Actuarial Science

13.45.1 Actuarial and Financial Tables

13.50 Humanities, Linguistics

13.55 Logic

14.00 Miscellaneous

14.05 Mathematical Research, Mathematical Education 


\section{INDEX OF REVIEWS BY AUTHOR OF WORK REVIEWED}

\begin{tabular}{|c|c|c|c|}
\hline Author & $\begin{array}{l}\text { Review } \\
\text { Vumber }\end{array}$ & Classification & Page \\
\hline ARSENIN, V. Y. & 21 & $\begin{array}{l}\text { See: TIKHONOV, A. N. \& ARSENIN, } \\
\text { V. Y. }\end{array}$ & 1320 \\
\hline ATKIN, A. O. L. & 5 & 9.00 & 315 \\
\hline BAILLIE, ROBERT & 29 & 9.00 & 1326 \\
\hline $\begin{array}{l}\text { BAJPAI, A. C., CALUS, I. M. \& } \\
\text { FAIRLEY, J. A. }\end{array}$ & 2 & $3.00,4.00$ & 311 \\
\hline $\begin{array}{l}\text { BEARD, JACOB T. B., JR. \& WEST, } \\
\text { KAREN I. }\end{array}$ & 25 & 10.00 & 1324 \\
\hline BRENT, RICHARD P. & 1 & $2.30,7.05$ & 311 \\
\hline CALUS, I. M. & 2 & $\begin{array}{l}\text { See: BAJPAI, A. C., CALUS, I. M. \& } \\
\text { FAIRLEY, J. A. }\end{array}$ & 311 \\
\hline $\begin{array}{l}\text { COLLATZ, L., MEINARDUS, G. \& } \\
\text { WETTERLING, W., Editors }\end{array}$ & 30 & 10.35 & 1327 \\
\hline DESCLOUX, J. \& MARTI, J., Editors & 27 & 2.00 & 1325 \\
\hline DEVITT, J.S. & 14 & 9.00 & 942 \\
\hline EDWARDS, HAROLD M. & 15 & 9.00 & 943 \\
\hline ERNVALL, REIJO & 11 & 9.00 & 656 \\
\hline FAIRLEY, J. A. & 2 & $\begin{array}{l}\text { See: BAJPAI, A. C., CALUS, I. M. \& } \\
\text { FAIRLEY, J. A. }\end{array}$ & 311 \\
\hline $\begin{array}{l}\text { FITZGIBBON, W. E. \& WALKER, } \\
\text { H. F., Editors }\end{array}$ & 23 & 5.00 & 1323 \\
\hline $\begin{array}{l}\text { GLOWINSKI, R., LIONS, J. L. \& } \\
\text { TRÉMOLIĖRES, R. }\end{array}$ & 4 & $5.00,6.30$ & 313 \\
\hline GOOS, G. \& HARTMANIS, J., Editors & 3 & 3.15 & 312 \\
\hline $\begin{array}{l}\text { GUTKNECHT, MARTIN, HENRICI, } \\
\text { PETER, LÄUCHLI, PETER \& } \\
\text { SCHWARZ, HANS-RUDOLF }\end{array}$ & 7 & $3.00,4.00$ & 651 \\
\hline HALL, G. \& WATT, J.M., Editors & 22 & $4.00,12.00$ & 1322 \\
\hline HARTMANIS, J. & 3 & See: GOOS, G. \& HARTMANIS, J., Editors & 312 \\
\hline HELLER, RALPH & 24 & 7.30 & 1324 \\
\hline HENRICI, PETER & 7 & $\begin{array}{l}\text { See: GUTKNECHT, MARTIN, HENRICI, } \\
\text { PETER, LÄUCHLI,PETER \& SCHWARZ } \\
\text { HANS-RUDOLF }\end{array}$ & 651 \\
\hline JACOBS, D. A.H., Editor & 26 & $2.05,2.10,3.00,4.00,5.00,6.15$ & 1325 \\
\hline $\begin{array}{l}\text { KARLIN, SAMUEL, MICCHELLI, } \\
\text { CHARLES A., PINKUS, ALLAN \& } \\
\text { SCHOENBERG, I. J. }\end{array}$ & 20 & 2.05 & 1318 \\
\hline LÄUCHLI, PETER & 7 & \multicolumn{2}{|l|}{$\begin{array}{l}\text { See: GUTKNECHT, MARTIN, HENRICI, } \\
\text { PETER, LÄUCHLI,PETER \& SCHWARZ, } \\
\text { HANS-RUDOLF }\end{array}$} \\
\hline LEECH, JOHN & 12 & 9.00 & 657 \\
\hline LEVINE, NORMAN & 13 & $\begin{array}{l}\text { See: SALZER, HERBERT E. \& LEVINE } \\
\text { NORMAN }\end{array}$ & 942 \\
\hline LIONS, J. L. & 4 & $\begin{array}{l}\text { See: GLOWINSKI, R., LIONS, J. L. \& } \\
\text { TRÉMOLIĖRES, R. }\end{array}$ & 313 \\
\hline MARTI, J. & 27 & See: DESCLOUX, J. \& MARTI, J., Editors & 1325 \\
\hline MEINARDUS, G. & 30 & $\begin{array}{l}\text { See: COLLATZ, L., MEINARDUS, G. \& } \\
\text { WETTERLING, W., Editors }\end{array}$ & 1327 \\
\hline MICCHELLI, CHARLES A. & 20 & $\begin{array}{l}\text { See: KARLIN, SAMUEL, MICCHELLI, } \\
\text { CHARLES A., PINKUS, ALLAN \& } \\
\text { SCHOENBERG, I. } .\end{array}$ & 1318 \\
\hline ODEN, J. T. \& REDDY, J. N. & 8 & $4.10 .4,5.10 .3,5.20 .4$ & 652 \\
\hline
\end{tabular}




\begin{tabular}{|c|c|c|c|}
\hline Author & $\begin{array}{l}\text { Review } \\
\text { Number }\end{array}$ & Classification & Page \\
\hline PINKUS, ALLAN & 20 & $\begin{array}{l}\text { See: KARLIN, SAMUEL, MICCHELLI, } \\
\text { CHARLES A., PINKUS, ALLAN \& } \\
\text { SCHOENBERG, I. J. }\end{array}$ & 1318 \\
\hline REDDY, J. N. & 8 & See: ODEN, J. T. \& REDDY, J. N. & 652 \\
\hline te RIELE, HERMAN J. J. & 16 & 9.00 & 944 \\
\hline te RIELE, HERMAN J. J. & 17 & 9.00 & 945 \\
\hline te RIELE, HERMAN J. $J$. & 18 & 9.00 & 945 \\
\hline $\begin{array}{l}\text { SALZER, HERBERT E. \& LEVINE, } \\
\text { NORMAN }\end{array}$ & 13 & (c) & 942 \\
\hline SCHOENBERG, I. J. & 20 & $\begin{array}{l}\text { See: KARLIN, SAMUEL, MICCHELLI, } \\
\text { CHARLES A., PINKUS, ALLAN \& } \\
\text { SCHOENBERG, I. J. }\end{array}$ & 1318 \\
\hline SCHWARZ, HANS-RUDOLF & 7 & $\begin{array}{l}\text { See: GUTKNECHT, MARTIN, HENRICI, } \\
\text { PETER, LÄUCHLI,PETER \& SCHWARZ } \\
\text { HANS-RUDOLF }\end{array}$ & ${ }^{Z}{ }_{651}$ \\
\hline SHALLIT, JEFFREY & 10 & $7.05,10.30$ & 656 \\
\hline SIGILLITO, V. G. & 9 & $5.10,5.20$ & 656 \\
\hline SPÄTH, HELMUTH & 19 & 2.05 & 1317 \\
\hline STETTER, HANS J. & 6 & $2.00,3.00,4.00$ & 651 \\
\hline TIKHONOV, A. N. \& ARSENIN, V.Y. & .21 & $3.10,6.15$ & 1320 \\
\hline TRÉMOLIĖRES, R. & 4 & $\begin{array}{l}\text { See: GLOWINSKI, R., LIONS, J. L. \& } \\
\text { TRÉMOLIĖRES, R. }\end{array}$ & 313 \\
\hline WALKER, H. F. & 23 & $\begin{array}{l}\text { See: FITZGIBBON, W. E. \& WALKER, H.F., } \\
\text { Editors }\end{array}$ & 1323 \\
\hline WATT, J. M. & 22 & See: HALL, G. \& WATT, J. M., Editors & 1322 \\
\hline WEST, KAREN I. & 25 & $\begin{array}{l}\text { See: BEARD, JACOB T. B., JR. \& WEST, } \\
\text { KAREN I. }\end{array}$ & 1324 \\
\hline WETTERLING, W. & 30 & $\begin{array}{l}\text { See: COLLATZ, L., MEINARDUS, G. \& } \\
\text { WETTERLING, W., Editors }\end{array}$ & \\
\hline & & & \\
\hline
\end{tabular}

INDEX OF REVIEWS BY SUBJECT OF WORK REVIEWED Author

Review

Number

2.00 Selected Topics in Numerical Analysis

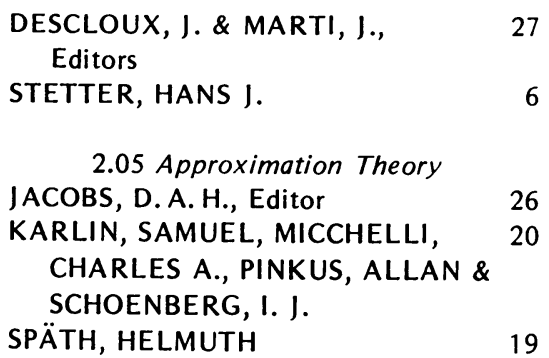

2.10 Numerical Integration JACOBS, D. A. H., Editor 2.30 Continued Fractions BRENT, RICHARD P.

3.00 Linear Algebra

BAJPAI, A. C., CALUS, I. M. \& FAIRLEY, J. A.

$$
\text { Title }
$$

Page

Numerical Analysis

Numerik für Informatiker-Computergerechte numerische Verfahren. Eine Einfuhrüng 651

The State of the Art in Numerical Analysis 1325 Studies in Spline Functions and Approximation Theory

Spline-Algorithmen zur Konstruktion glatter Kurven und Flächen

The State of the Art in Numerical Analysis 1325

$\gamma$ and $e^{\gamma}$ to $20700 \mathrm{D}$ and Their Regular Continued Fractions to 20000 Partial Quotients 
Author $\begin{array}{r}\text { Review } \\ \text { Number }\end{array}$

GUTKNECHT, MARTIN, HENRICI, PETER, LÄUCHLI, PETER \& SCHWARZ, HANS-RUDOLF

JACOBS, D.A.H., Editor

STETTER, HANS J.

3.10 Linear Equations

TIKHONOV, A. N. \& ARSENIN, V. Y. 21

3.15 Eigenvalues and Eigenvectors

GOOS, G. \& HARTMANIS, J., Editors 3

4.00 Ordinary Differential Equations

BAJPAI, A. C., CALUS, I. M. \& 2

FAIRLEY, J. A.

GUTKNECHT, MARTIN, HENRICI, 7 PETER, LÄUCHLI, PETER \&

SCHWARZ, HANS-RUDOLF

HALL, G. \& WATT, J. M., Editors 22

JACOBS, D. A. H., Editor 26

STETTER, HANS J.

4.10.4 Finite Element Methods

ODEN, J. T. \& REDDY, J. N.

5.00 Partial Differential Equations

FITZGIBBON, W. E. \& WALKER, 23 H. F., Editors

GLOWINSKI, R., LIONS, J. L. \& 4 TRÉMOLIÈRES, R.

JACOBS, D. A. H., Editor

5.10 Boundary Value Problems

SIGILLITO, V. G.

5.10.3 Finite Element and Other $\begin{array}{lc}\text { Title } & \text { Page } \\ \text { Heinz Rutishauser: Vorlesungen über } & \\ \text { numerische Mathematik, Vols. } 1 \text { and } 2 & 651 \\ \text { The State of the Art in Numerical Analysis } & 1325 \\ \text { Numerik für Informatiker-Computergerechte } \\ \text { numerische Verfahren. Eine Einführung }\end{array}$

Numerical Methods for Engineers and Scientists

Heinz Rutishauser: Vorlesungen über numerische Mathematik, Vols. 1 and 2

Modern Numerical Methods for Ordinary Differential Equations

The State of the Art in Numerical Analysis 1325

Numerik für Informatiker-Computergerechte numerische Verfahren. Eine Einführung 651

An Introduction to the Mathematical Theory of Finite Elements

Nonlinear Diffusion

1323

Analyse Numérique de Inéquations Variationnelles, Vol. 1; Applications aux Phénomènes Stationnaires et d'Évolution, Vol. $2 \quad 313$

The State of the Art in Numerical Analysis 1325

9 Explicit A Priori Inequalities With Applications to Boundary Value Problems 656

Approximation Methods

ODEN, J. T. \& REDDY, J. N. $\quad 8 \quad$ An Introduction to the Mathematical Theory of Finite Elements 652

5.20 Mixed Initial and Boundary Value Problems

SIGILLITO, V. G.

9 Explicit A Priori Inequalities With Applications to Boundary Value Problems

656

5.20.4 Finite Element and Other Approximation Methods

ODEN, J. T. \& REDDY, J. N.

8

An Introduction to the Mathematical Theory of Finite Elements

6.00 Other Functional Equations

6.15 Integral Equations

JACOBS, D. A. H., Editor 26

TIKHONOV, A. N. \& ARSENIN, V.Y. 21 6.30 Variational Equations

GLOWINSKI, R., LIONS, J. L. \& 4 TRÉMOLIĖRES, R.
The State of the Art in Numerical Analysis 1325

Solutions of III-Posed Problems

1320

Analyse Numérique de Inéquations Variationnelles, Vol. 1; Applications aux Phénomènes Stationnaires et d'Évolution, Vol. 2 


$$
\begin{array}{ccc}
\text { Review } & \text { Tithor } & \text { Number }
\end{array}
$$

7.00 Special Functions

7.05 Mathematical Constants, Special Polynomials (Nonorthogonal) and Numbers: Bernoulli, Euler, Stirling, Binomial Coefficients, Factorials

BRENT, RICHARD P.

SHALLIT, JEFFREY

7.30 Bessel Functions

HELLER, RALPH
$1 \gamma$ and $e^{\gamma}$ to $20700 \mathrm{D}$ and Their Regular Continued Fractions to 20000 Partial Quotients

10 Table of Bell Numbers to $B(400)$ 311 656

$2425 \mathrm{D}$ Table of the First One Hundred Values of $j_{0, s}, J_{1}\left(j_{0, s}\right), j_{1, s}, J_{0}\left(j_{1, s}\right)=J_{0}\left(i_{0, s+1}^{\prime}\right)$ $j_{1, s,}^{\prime} \dot{J}_{1}\left(j_{1}^{\prime}, s\right)$

7.55 Expansions in Series of Orthogonal Polynomials, Bessel Functions, Other Functions SALZER, HERBERT E. \& LEVINE, 13 NORMAN

Tables of $2^{-r+1}{ }_{r} C(r-k) / 2$

9.00 Number Theory

\begin{tabular}{|c|c|c|}
\hline ATKIN, A. O. L. & 5 & $\begin{array}{l}\text { The Number of Subgroups of the Classical } \\
\text { Modular Group of Index } N\end{array}$ \\
\hline BAILLIE, ROBERT & 29 & $\begin{array}{l}\text { Solutions of } \varphi(n)=\varphi(n+1) \text { for Euler's } \\
\quad \text { Function }\end{array}$ \\
\hline DEVITT, J. S. & 14 & Some Tables for Aliquot Sequences \\
\hline EDWARDS, HAROLD M. & 15 & $\begin{array}{l}\text { Fermat's Last Theorem. A Genetic Intro- } \\
\text { duction to Algebraic Number Theory }\end{array}$ \\
\hline ERNVALL, REIJO & 11 & E-Irregular Primes and Related Tables \\
\hline LEECH, JOHN & 12 & Five Tables Relating to Rational Cuboids \\
\hline te RIELE, HERMAN J. J. & 16 & Unitary Aliquot Sequences \\
\hline te RIELE, HERMANN J. J. & 17 & $\begin{array}{l}\text { Further Results on Unitary Aliquot } \\
\text { Sequences }\end{array}$ \\
\hline te RIELE, HERMAN J. J. & 18 & $\begin{array}{l}\text { A Theoretical and Computational Study of } \\
\text { Generalized Aliquot Sequences }\end{array}$ \\
\hline \multicolumn{3}{|c|}{ 10.00 Algebra and Combinatorial Theory } \\
\hline $\begin{array}{l}\text { BEARD, JACOB T. B., JR. \& WEST, } \\
\text { KAREN I. }\end{array}$ & 25 & $\begin{array}{l}\text { Factorization Tables for Binomials over } \\
\qquad \mathrm{GF}(q)\end{array}$ \\
\hline \multicolumn{3}{|c|}{10.30 Permutations, Combinatorial Identities } \\
\hline $\begin{array}{l}\text { SHALLIT, JEFFREY } \\
10.35 \text { Graph Theory }\end{array}$ & 10 & Table of Bell Numbers to $B(400)$ \\
\hline $\begin{array}{l}\text { COLLATZ, L., MEINARDUS, G. \& } \\
\text { WETTERLING, W., Editors }\end{array}$ & 30 & $\begin{array}{l}\text { Numerische Methoden bei Optimierungsauf- } \\
\text { gaben, Band } 3 \text {, Optimierung bei graphen- } \\
\text { theoretischen und genzzahliger } \\
\text { Problemen }\end{array}$ \\
\hline ZWILLINGER, DAN & 28 & Magic Labellings \\
\hline
\end{tabular}

1326

942

943

656

657

944

945

945

12.00 Computers and Other Aids to Computation

HALL, G. \& WATT, J. M., Editors 22 Modern Numerical Methods for Ordinary Differential Equations

1322

\section{INDEX OF TABLE ERRATA}

No. Author

Title

Page

548 ABRAMOWITZ, MILTON \& STEGUN, IRENE A., Editors

Handbook of Mathematical Functions with Formulas, Graphs, and Mathematical Tables

549 BEYER, W. A. \& WATERMAN, M. S.

Decimals and Partial Quotients of Euler's Constant and In 2

Tables of Integrals, Series, and Products

A Treatise on Bessel Functions and Their
551 GRAY, A., MATHEWS, G. B. \& MACROBERT, T. M.
Applications to Physics 
552 HART, JOHN F., CHENEY, E. W.,

LAWSON, CHARLES L., MAEHLY,

HANS J., MESZTENYI, CHARLES K.,

RICE, JOHN R., THACHER, HENRY

G., JR. \& WITZGALL, CHRISTOPH

554 KRAITCHIK, M.

555 KRAITCHIK, M.

553 MAGNUS, W., OBERHETTINGER, F. \& SONI, R. P.
Théorie des Nombres, t. 3, Analyse Diophantine et Applications aux Cuboides Rationnels 661

Sur les Cuboides Rationnels 661

Formulas and Theorems for the Special Functions of Mathematical Physics

\section{INDEX OF CORRIGENDA}

Author

JELTSCH, ROLF

JELTSCH, ROLF

SHANKS, DANIEL
Title

Page

Taylor Series Coefficients of the Jacobian Functions by Alois Schett

Multistep Methods Using Higher Derivatives and Damping at Infinity by Rolf Jeltsch 1328

Gauss's Ternary Form Reduction and the 2-Sylow Subgroup

\section{INDEX OF MICROFICHE SUPPLEMENTS}

Author

BABUŠ́KA, I. \& OSBORN, J. E. JOHNSON, J. H.

CHIN, R.C.Y \& HEDSTROM, G. W.

KISHORE, MASAO

LEHMER, D. H. \& MASLEY, J. M.
BLAIR, J. M., EDWARDS, C. A. \&

Title

MOC Issue

Numerical Treatment of Eigenvalue Problems for Differential Equations with Discontinuous Coefficients

Rational Chebyshev Approximations for the Bickley Functions $K i_{n}(x)$

October

A Dispersion Analysis for Difference Schemes: Tables of Generalized Airy Functions

October

Odd Integers $N$ With Five Distinct Prime Factors for Which $2-10^{-12<}$ $\sigma(N) / N<2+10^{-12}$

January

Table of the Cyclotomic Class Numbers $h^{*}(p)$ and Their Factors for $200<p$ $<521$

April and October

PEXTON, ROBERT L. \& STEIGER, ARNO D. Roots of Two Transcendental Equations as Functions of a Continuous Real

Parameter 